\title{
ÍNDICE DE PUBLICACIÓN SEGÚN ORIGEN DE LOS ARTÍCULOS DE LA REVISTA CHILENA DE CIRUGÍA. EVALUACIÓN PERÍODO 2007-2012*
}

\author{
Drs. Javier Moraga C. ${ }^{1,2}$, Ricardo Cartes-Velásquez ${ }^{3,4}$, Carlos Manterola D. ${ }^{2,3,5}$, \\ Sebastián Urrutia V.5. Grupo MINCIR (Metodología e Investigación en CIRugía).
}

1 Universidad Autónoma de Chile. Santiago.

2 Programa de Magíster en Ciencias Médicas, Mención Cirugía, Universidad de La Frontera. Temuco.

3 Programa de Doctorado en Ciencias Médicas, Universidad de La Frontera. Temuco.

${ }^{4}$ Facultad de Odontología, Universidad de Concepción, Concepción.

5 Departamento de Cirugía y Traumatología. Facultad de Medicina, Universidad de La Frontera. Temuco. Chile.

\begin{abstract}
Rate of publishing according to origin of the articles in Revista Chilena de Cirugia. Assessment of the period 2007-2012

Background: One of the variables analyzed in bibliometric studies is the origin of the publications, making it possible to quantify the impact of different universities, medical centers and Regions in the number of publications of a particular journal. The aim of this study is to determine the rate of publication of the "Revista Chilena de Cirugía" (RCC) in the period 2007-2012 and its relationship to origin (Regions and Chapters of the Society of Surgeons of Chile) and university affiliation (UA). Material and Methods: Bibliometric study. All the RCC numbers between 2007 and 2012 were analyzed. The articles were categorized according to year of publication, article type, country Region that originated the article and UA expressed in every article. We determined the percentage of UA in each Region, we calculated the rate of publication, the overall publication average and the average annual rate (AAR). Results: In the study period 687 articles were published, with an average of 114.5 and 19.1 articles per volume and number respectively; of these, 602 met the selection criteria. 60.6\%; 8.6\% and 5\% were generated in Regions Metropolitana, Araucanía and Biobío respectively. The $75.4 \%$ of the papers presented UA. The AAR was $1.1 \pm 0.7$ publications/year x 10 members, being higher for Chapters IX and IV Regions with $2.3 \pm 1,0$ and $1.7 \pm 0.5$ publications/year x 10 members respectively. Conclusion: Most of the articles are generated in Metropolitana Region. A noticeable percentage of publications show no UA. Most AAR has been verified in regions.
\end{abstract}

Key words: "Bibliometrics" [MeSH], Surgery, Chile, Regions.

*Recibido el 19 de diciembre de 2013 y aceptado para publicación el 25 de marzo de 2014.

Los autores declaran no tener conflicto de interés.

Correspondencia: Dr. Javier Moraga C.

Javier.moragac@gmail.com 


\section{Resumen}

Introducción: Una de las variables que se analizan en los estudios bibliométricos es la procedencia de las publicaciones, lo que ha permitido cuantificar el impacto que tienen las distintas universidades, centros asistenciales y regiones en el total de publicaciones de una revista en particular. El objetivo de este estudio es determinar el índice de publicación de la Revista Chilena de Cirugía (RCC) en el período 2007-2012 y su relación con procedencia (Regiones y Capítulos de la Sociedad de Cirujanos de Chile) y filiación universitaria (FU). Material y Método: Estudio bibliométrico. Se analizaron todos los números de la RCC entre los años 2007 y 2012. Los artículos se categorizaron de acuerdo al año de publicación, tipo de artículo, Región del país donde se originó el artículo y FU manifestada en cada artículo. Se determinó el porcentaje de FU de cada Región, se calculó la tasa de publicación, el promedio general de publicación y la tasa de publicación anual promedio (TPAP). Resultados: En el período estudiado se publicaron 687 artículos; con un promedio de 114,5 y 19,1 artículos por volumen y por número respectivamente; de los cuales, 602 cumplían con los criterios de selección. El 60,6\%, 8,6\% y 5\% fueron generados en las Regiones Metropolitana, de la Araucanía y del Biobío respectivamente. El 75,4\% de las publicaciones presentó FU. La TPAP fue de 1,1 \pm 0,7 publicaciones/año x 10 miembros, siendo mayor para los Capítulos de la IX y IV Regiones con un 2,3 $\pm 1,0$ y un $1,7 \pm 0,5$ publicaciones/año x 10 miembros respectivamente. Conclusiones: La mayor parte de los artículos se generan en la Región Metropolitana. Un porcentaje considerable de las publicaciones no manifiesta FU. La mayor TPAP se verificó en Regiones.

Palabras clave: Bibliometría, cirugía, Chile, regiones.

\section{Introducción}

Los análisis bibliométricos nacen como una herramienta de medición de la literatura que permite observar desde un punto de vista crítico el desarrollo de la actividad científica en diversas áreas ${ }^{1}$. Asimismo, estos análisis permiten caracterizar la producción científica de personas, instituciones y naciones, tanto en términos cuantitativos como cualitativos ${ }^{2,3}$.

En los estudios bibliométricos clásicos la medida más utilizada es el factor de impacto ${ }^{1,4-7}$. Sin embargo, existen otras variables de interés como son productividad, visibilidad ${ }^{7-8}$, calidad metodológica ${ }^{9-12}$, procedencia $^{1}$ y filiación a centros académicos ${ }^{10}$, entre otros.

En esta línea de investigación, en 2008 se publicó un estudio bibliométrico en el que se valoró la procedencia de los artículos publicados en la Revista Chilena de Cirugía (RCC). En aquel momento se incluyeron todos los artículos que se habían publicado entre los años 2002 y 2006 (ambos incluidos); y se constató que de los 545 artículos analizados el $67,9 \%$ fue generado en la Región Metropolitana; el $10,8 \%$ en la Región del Biobío; y el 7,8\% en la Región de la Araucanía. Sin embargo, al analizar la tasa de publicación anual (artículos por cada 10 cirujanos miembros de la Sociedad de Cirugía de Chile [SCCh]); esta fue mayor en Regiones, destacando un $2,8 \pm 0,8$ y un $1,8 \pm 0,2$ publicaciones/año x 10 miembros, para los Capítulos de la IX y VIII Región respectivamente; con un $64,8 \%$ de filiación a centros académicos del total de las publicaciones del período ${ }^{10}$.

Aunque la metodología aplicada para la reali- zación de este tipo de análisis puede resultar controversial; este tipo de mediciones constituye un elemento trascendente para valorar el nivel científico de los grupos de trabajos, instituciones y revistas ${ }^{13}$. Es por esto que nos parece pertinente realizar análisis periódicos de la realidad de nuestra revista; en este caso, evaluando el índice de publicación según procedencia.

El objetivo de este estudio es determinar el índice de publicación de la RCC en el período 2007-2012 y su relación con procedencia (Regiones y Capítulos de la SCCh) y filiación universitaria (FU).

\section{Material y Método}

\section{Diseño}

Estudio bibliométrico.

\section{Población a estudio}

Todos los artículos publicados en la RCC entre los años 2007 y 2012 (ambos incluidos) $)^{14}$.

\section{Criterios de selección}

Criterios de inclusión: Artículos publicados en la RCC entre los años 2007 y 2012 ambos incluidos, sin restricción de diseño, origen ni área temática.

Criterios de exclusión: Artículos publicados en las secciones Revista de Revistas, Crónicas, Obituario, Maestros de la cirugía, Cartas al editor.

\section{Maniobra}

Se realizó una revisión de todos los números de la $\mathrm{RCC}$ en el período antes señalado. Se categorizaron 
los artículos de acuerdo al año de publicación, tipo de artículo (editorial, artículos de investigación, casos clínicos, cirugía al día, técnica quirúrgica, documentos, imágenes y cirugía, artículos de revisión); la Región del país en la que se originó el artículo y la FU manifestada en el artículo. Se revisaron los archivos de la SCCh para identificar el número total de miembros por Capítulo y se emparejó cada $\mathrm{Ca}$ pítulo con su Región correspondiente, exceptuando el Capítulo X, XI y XIV que incluye miembros provenientes de las tres Regiones. En este caso, se sumaron los artículos generados por las tres Regiones.

\section{Definiciones}

Tasa de publicación (TP): Razón que se obtiene entre el total de publicaciones de un período de tiempo generado por un grupo de personas, por el total de personas. En este estudio se definió a cada Capítulo de la SCCh como un grupo de personas capaces de generar artículos. Debido al reducido número de socios que componen cada Capítulo se decidió ajustar este índice por 10, de modo tal, de obtener un número fácil de interpretar ${ }^{1}$.

\section{Tasa publicación anual $(T P A)=$ $N^{\circ}$ publicaciones año " $X$ ” $x 10$ $N^{\circ}$ miembros Capítulo "Y” SCCh}

$$
T P=
$$

TPA(2007)+TPA(2008)+TPA(2009)+ TPA(2010)+TPA(2011)+TPA(2012)

\section{$N^{\circ}$ de años en estudio (6)}

Promedio general de publicación (PGP): Cuociente entre el número de publicaciones generadas en el período en estudio y el total de volúmenes de la revista estudiados (se estudiaron 6 volúmenes).

$$
P G P=
$$

Publicaciones periodo en estudio

$N^{\circ}$ volúmenes estudiados (6)

Tasa se publicación anual promedio (TPAP): Promedio de las tasas de publicación presentada por cada Capítulo de la SCCh.

\section{$T P A P=$}

TP(Cap.II $)+T P($ Cap.IV $)+T P($ Cap.V $)+$ TP(Cap.RM $)+T P($ Cap.VIII $)+T P($ Cap.IX $)+$ TP(Cap.X-XI-XIV) $N^{\circ}$ de Capitulos (7)
Tabla 1. Capítulos de la SCCh y su número de miembros

\begin{tabular}{|cc|}
\hline Capítulo & n Miembros \\
II & 14 \\
IV & 21 \\
V & 79 \\
RM & 538 \\
VIII & 50 \\
IX & 37 \\
X-XI-XIV & 35 \\
Total & 774 \\
\hline
\end{tabular}

\section{Plan de análisis}

Se realizó un análisis exploratorio de los datos y se aplicó estadística descriptiva, determinando el porcentaje de FU de cada Región; y calculando la TP y PGP con sus respectivas desviaciones estándar; y la TPAP.

\section{Resultados}

Durante el período en estudio se publicaron 6 volúmenes y 36 números de la RCC, con un total de 687 artículos; con un promedio de 114,5 y 19,1 artículos por volumen y por número respectivamente. El número de miembros de la $\mathrm{SCCh}$ y de sus respectivos Capítulos se detalla en la Tabla 1.

Del total de los artículos publicados; 602 cumplían con los criterios de selección. De estos; el $60,6 \%, 8,6 \%$ y $5 \%$ fueron generados en las Regiones Metropolitana, de la Araucanía y del Biobío respectivamente (Tabla 2). El 85,4\% de las publicaciones corresponden a las Regiones que representan a los distintos Capítulos de la SCCh (Capítulos II, IV, V, VIII, IX, X, XI, XIV y RM). El detalle de las publicaciones por Capítulo de la SCCh se observa en la Tabla 3. El 75,4\% de las publicaciones presentó FU, destacando entre estas la Universidad de Chile, Pontificia Universidad Católica de Chile y Universidad de La Frontera con un 37,0\%, 17,8\% y 11,2\% respectivamente del total de publicaciones con $\mathrm{FU}$ (Tabla 4).

La TPAP fue de $1,1 \pm 0,7$ publicaciones/año $\mathrm{x}$ 10 miembros; siendo mayor para los Capítulos de la IX y IV Regiones con un $2,3 \pm 1,0$ y un $1,7 \pm 0,5$ publicaciones/año x 10 miembros respectivamente (Tabla 2). 
ÍNDICE DE PUBLICACIÓN SEGÚN ORIGEN DE LOS ARTÍCULOS DE LA REVISTA CHILENA DE CIRUGÍA...

Tabla 2. Publicaciones según Región del país

\begin{tabular}{|c|c|c|c|c|}
\hline $\begin{array}{l}\text { Origen de la } \\
\text { publicación }\end{array}$ & $\begin{array}{c}\text { Publicaciones } \\
\text { n } \quad(\%)\end{array}$ & $\begin{array}{c}\text { Filiación universitaria } \\
(\%)\end{array}$ & $\begin{array}{l}\text { Promedio general de } \\
\text { publicaciones }\end{array}$ & $\mathbf{T P A P} \pm \mathbf{D S}$ \\
\hline I & $0 \quad(0,0)$ & 0,0 & 0,0 & - \\
\hline II & $0 \quad(0,0)$ & 0,0 & 0,0 & - \\
\hline III & $(0,0)$ & 0,0 & 0,0 & - \\
\hline IV & $21 \quad(3,5)$ & 76,2 & 3,5 & $1,7 \pm 0,5$ \\
\hline V & $23 \quad(3,8)$ & 78,3 & 3,8 & $0,5 \pm 0,2$ \\
\hline $\mathrm{RM}$ & $365 \quad(60,6)$ & 81,1 & 60,8 & $1,1 \pm 1,1$ \\
\hline VI & $1 \quad(0,2)$ & 0,0 & 0,2 & - \\
\hline VII & $8 \quad(1,3)$ & 62,5 & 1,3 & - \\
\hline VIII & $30 \quad(5,0)$ & 100,0 & 5,0 & $1,0 \pm 0,8$ \\
\hline IX & $52 \quad(8,6)$ & 100,0 & 8,7 & $2,3 \pm 1,0$ \\
\hline X- XI- XIV & $24 \quad$ (4) & 75,0 & 4,0 & $1,1 \pm 0,4$ \\
\hline XII & $0 \quad(0,0)$ & 0,0 & 0,0 & - \\
\hline XV & $0 \quad(0,0)$ & 0,0 & 0,0 & - \\
\hline Extranjero & $78 \quad(13,0)$ & 24,3 & 13,0 & - \\
\hline Total & $602(100,0)$ & 75,4 & 96,8 & \\
\hline
\end{tabular}

Estimado con base en relación al número registrado de miembros de cada Capítulo de la SCCh. Al 11 de marzo de 2013. DS $=$ Desviación estándar.

Tabla 3. Publicaciones según Capítulo de la SCCH

\begin{tabular}{|c|c|c|c|c|c|}
\hline Capítulo & $\begin{array}{c}\text { Publ } \\
\text { n }\end{array}$ & $\begin{array}{l}\text { aciones } \\
(\%)\end{array}$ & $\begin{array}{c}\text { Filiación universitaria } \\
(\%)\end{array}$ & $\begin{array}{l}\text { Promedio general de } \\
\text { publicaciones }\end{array}$ & $\mathbf{T P A P} \pm \mathrm{DS}$ \\
\hline II & 0 & $(0,0)$ & 0,0 & 0,0 & \\
\hline IV & 21 & $(3,5)$ & 76,2 & 3,5 & $1,7 \pm 0,5$ \\
\hline V & 23 & $(3,8)$ & 78,3 & 3,8 & $0,5 \pm 0,2$ \\
\hline $\mathrm{RM}$ & 365 & $(60,6)$ & 81,1 & 60,8 & $1,1 \pm 1,1$ \\
\hline VIII & 30 & $(5,0)$ & 100,0 & 5,0 & $1,0 \pm 0,8$ \\
\hline IX & 52 & $(8,6)$ & 100,0 & 8,7 & $2,3 \pm 1$ \\
\hline X-XI-XIV & 24 & $(4,0)$ & 75,0 & 4,0 & $1,1 \pm 0,4$ \\
\hline Otros & & 87,0 & 27,6 & 14,5 & - \\
\hline Total & 602 & & 75,4 & - & - \\
\hline
\end{tabular}

DS= Desviación estándar. 
Tabla 4. Publicaciones según FU

\begin{tabular}{|lrc|}
\hline Universidad & n & \% \\
Universidad de Chile & 168 & 37 \\
Pontificia Universidad Católica de Chile & 81 & 17,8 \\
Universidad de La Frontera & 51 & 11,2 \\
Universidad de Concepción & 23 & 5,1 \\
Universidad Austral de Chile & 18 & 4,0 \\
Universidad de Valparaíso & 18 & 4,0 \\
Universidad Católica del Norte & 16 & 3,5 \\
Universidad de los Andes & 16 & 3,5 \\
Universidad Andrés Bello & 16 & 3,5 \\
Otras* & 47 & 10,4 \\
Total & 454 & 100 \\
\hline
\end{tabular}

*Universidad Católica de la Santísima Concepción, Universidad Católica del Maule, Universidad Mayor, Universidad de Santiago de Chile, Universidad del Desarrollo, Universidad Diego Portales, Universidad de Talca, Universidad San Sebastián, Universidades extranjeras.

\section{Discusión}

Este estudio se realizó por el interés de generar análisis periódicos a nivel de la RCC, respecto de las variables antes descritas; de tal forma de conocer la productividad de centros, Capítulos y Regiones; hecho que puede permitir a través de la actividad societaria, realizar intervenciones para incrementar no sólo la productividad sino también la calidad metodológica de lo que se publica en la RCC.

$\mathrm{Al}$ analizar los resultados de esta investigación podemos observar que el mayor porcentaje de artículos se genera en la Región Metropolitana (60,6\%); lo que se condice con el resultado obtenido en la medición anteriormente publicada. Sin embargo, llama la atención la productividad del Capítulo de la segunda Región que no presenta productividad en el período en estudio. Otro hecho interesante de comentar es el aumento de más de 6 veces de las publicaciones extranjeras, de un $2,0 \%$ a un $13,0 \%$; situación posiblemente atribuible al aumento de la visibilidad internacional de la RCC posterior a su indización en la base de datos Science Citation Index Expanded de Thomson Reuters en $2009^{15}$.

En el sentido inverso podemos observar un descenso de las publicaciones de las regiones ligadas a los diferentes Capítulos de la SCCh de un 91,8\% a un $85,4 \%$, misma situación evidenciada en las regiones que no se encuentran ligadas a un Capítulo en particular; lo que puede asociarse al incremento de las publicaciones provenientes de centros extranjeros.

$\mathrm{Al}$ analizar los resultados ajustados por el número de cirujanos que componen cada Capítulo (TPAP); observamos que la productividad es mayor en regiones, específicamente en la Región de la Araucanía y Coquimbo; situación similar a la registrada en la medición anterior.

Otro hecho que se pone de manifiesto es el aumento de la FU durante este período de un 64,8\% a un $75,4 \%$; lo que presenta una cierta lógica, ya que por definición son los centros académicos los llamados a generar el conocimiento.

A pesar de las limitaciones propias de un estudio bibliométrico, esperamos que éste, sea de utilidad para la SCCh y cada uno de sus miembros en particular; para poder tomar acciones en la línea de incrementar la productividad científica de los Capítulos, los miembros de nuestra Sociedad y de los cirujanos chilenos en general.

\section{Referencias}

1. Vial M, Manterola C, Pineda V. Revista Chilena de Cirugía: Índice de publicación según procedencia de los artículos. Rev Chil Cir. 2008;60:41-5.

2. Abramo G, Ciriaco A, Di Costa F. National research assessment exercises: a comparison of peer review and bibliometrics rankings. Scientometrics 2011. En prensa.

3. Poletto VC, Faraco IM. Bibliometric study of articles published in a Brazilian journal of pediatric dentistry. Braz Oral Res. 2010;24:83-8.

4. Manterola C. Respecto de la calidad metodológica de los artículos que se publican en las revistas biomédicas. Rev Chil Cir. 2005;57:449-50.

5. Manterola C, Pineda V, Vial M, Losada H. ¿Es el factor de impacto un índice apropiado para determinar el grado de evidencia de estudios sobre procedimientos terapéuticos en revistas quirúrgicas? Cir Esp. 2005;78:96-9.

6. Chattopadhyay A. How useful is journal impact factor? Indian J Dent Res. 2009;20:246-8.

7. Moraga J, Cartes-Velásquez R, Manterola C, Urrutia $\mathrm{S}$. Publicaciones de autores chilenos en revistas quirúrgicas durante los últimos diez años. Rev Chil Cir. 2012;64:447-51.

8. Cartes-Velásquez R, Moraga J, Aravena P, Manterola C. Impacto y visibilidad de la Revista Chilena de Cirugía tras su indización en las bases de dato SciELO e ISI. Análisis Bibliométrico. Rev Chil Cir. 2012;64:511-15.

9. Pineda V, Manterola C, Vial M, Losada H. ¿Cuál es la calidad metodológica de los artículos referentes a terapia, publicados en la revista chilena de cirugía? Rev Chil Cir. 2005;57:500-7.

10. Manterola C, Busquets J, Pascual M, Grande L. ¿Cuál es la calidad metodológica de los artículos sobre proce- 
dimientos terapéuticos publicados en Cirugía Española? Cir Esp. 2006;79:95-100.

11. Manterola C, Pineda V, Vial M, Losada H. The MINCIR Group. What is the Methodologic Quality of human therapy studies in ISI surgical publications? Ann Surg. 2006;244:827-32.

12. Manterola C, Grande L. Calidad metodológica de los artículos publicados en Cirugía Española referentes a procedimientos terapéuticos. Evaluación del período 2005- 2008. Cir Esp. 2010;87:244-50.

13. Garfield E. Fortnightly review: How can impact factors be improved? Br Med J. 1996;313:411-3.

14. www.scielo.cl/scielo.php?pid=0718-4026\&script $=$ sci issues. Visitado el 14 de febrero de 2013.

15. Arribalzaga E. Incorporación al ISI de la Revista Chilena de Cirugía. Rev Chil Cir. 2009;61:313. 